\title{
Stabilized Procedures for Finite Element Analysis in Saturated Soils Under Cyclic Loading
}

\author{
Maosong Huang ${ }^{1}$, Ming Liu ${ }^{1}$, and O.C. Zienkiewicz ${ }^{2}$ \\ ${ }^{1}$ Department of Geotechnical Engineering, Tongji University, 200092 Shanghai, China \\ ${ }^{2}$ Institute for Numerical Methods in Engineering, University of Wales Swansea, \\ Swansea SA2 8PP, U.K. \\ mshuang@mail..tongji.edu.cn
}

\begin{abstract}
Conventional finite element procedures with equal order interpolations for saturated soil dynamic $\boldsymbol{u}-p$ formulation have been experiencing numerical difficulties for the problems concerning with small permeability and neglectable compressibility of pore-fluid. Such difficulties can be avoided by the staggered implicit-implicit algorithm and the direct $\alpha$ method which were introduced by Huang et al. (2004). Several numerical examples for surface step loadings were given to demonstrate the performance of the proposed methods. Since the numerical examples for the step loadings are not typical for dynamic problems, which usually show the relatively strong oscillation nature of dynamic behaviors. Due to the necessity to show the effectiveness of the proposed stabilization techniques for cyclic loading problems, two numerical examples regarding saturated soils subject to a surface cyclic loading are presented in this paper. Discussion on uniform and nonuniform mesh configuration is also given.
\end{abstract}

Keywords: Saturated soils; Cyclic loading; $\boldsymbol{u}$ - $p$ formulation; Incompressible behavior; Stabilization procedures.

\section{Introduction}

Extensive research has been devoted during the past few decades to coupled dynamic analysis of soil - pore fluid interaction problems due to its application to the problems subjected to earthquake or other types of cyclic loadings. Verification exercises assisted by the VELACS (VErification of Liquefaction Analysis by Centrifuge Studies) project of the US National Science Foundation have demonstrated the importance of the full two-phase, numerical analysis for saturated soil dynamic problems. The problems are generally formulated in terms of the displacement of the solid phase $\boldsymbol{u}$ and the pore-water pressure $p$ under certain assumptions ${ }^{[1-2]}$. The numerical solution procedure for solving such a $\boldsymbol{u}-p$ formulation with a general direct implicit time-stepping algorithm was discussed fully in Ref. [3]. It requires that the interpolation functions for $\boldsymbol{u}$ and $p$ in the finite element discretization must fulfill the Babuska-Brezzi conditions or the much simpler Zienkiewicz-Taylor patch test in the limit of nearly incompressible pore fluid and small permeability. Only certain combinations of shape functions for $u$ and $p$ variables are permissible for the mixed 
formulation. In particular, when both the permeability and compressibility of the pore fluid tend to zero, the solution will 'lock' or oscillate wildly unless the displacements are interpolated from their nodal values by polynomials one order higher than those used for the pressure. The problem here is identical to that encountered in solid mechanics or fluid mechanics. The restriction of mixed formulation will complicate the finite element coding and preclude the use of otherwise useful element types. Therefore, it is necessary to introduce simple stabilization techniques which permit continuous and equal interpolation to be used for $\boldsymbol{u}$ and $p$.

Three classes of the stabilization methods have been introduced in the literature so far. The first of them is referred to as the fractional step method or operator-splitting method, which can be realized in either semi-explicit form or staggered implicitimplicit form ${ }^{[4-5]}$. Advantages of the introduction of the staggered implicit-implicit form over the semi-explicit form are not just its unconditional stability. Meanwhile, optimal solutions can be achieved with the staggered implicit-implicit form. As already explained in Ref. [6], the proposed fractional step method (or staggered algorithm) may experience difficulties due to the time-stepping stability requirement. Such difficulties can be partially overcome with the introduction of the second class of the stabilization techniques, which is so-called direct $\alpha$-method ${ }^{[6]}$. As an alternative, Mira et al. ${ }^{[7]}$ presented a new formulation in which stabilization was achieved through an approach based on the enhanced strain element. However, only the problems of quasi-static consolidation were discussed in Ref. [7]. Further research the application of the proposed method into saturated soil dynamic problems is obviously required. Indeed, the analysis of examples with surface step loadings used by the authors in Ref. [6] is not quite typical for dynamic problems, since numerical errors caused by numerical stability problems may be gradually accumulated for the examples with cyclic loadings. Therefore, in this paper several numerical examples subjected to cyclic loadings are provided to demonstrate numerical stability of the previously proposed techniques for dynamic problems.

\section{Governing Equations and Stabilized Procedures}

Here the staggered implicit-implicit algorithm and the direct $\alpha$-method proposed by the authors ${ }^{[5-6]}$ are briefly reviewed. As described in Ref. [6], the governing total momentum equilibrium and conservation equation of pore-fluid flow for $\boldsymbol{u}-p$ formulation can be written as

$$
\begin{gathered}
\boldsymbol{S}^{T}\left(\boldsymbol{\sigma}^{\prime}-\boldsymbol{m} p\right)+\rho \boldsymbol{b}-\boldsymbol{\rho} \ddot{\boldsymbol{u}}=0 . \\
\boldsymbol{m}^{T} \boldsymbol{S} \dot{\boldsymbol{u}}-\nabla^{T} \bar{k} \nabla p+\frac{n}{K_{f}} \dot{p}+\nabla^{T} \bar{k} \rho_{f} \boldsymbol{b}=0 .
\end{gathered}
$$

where $\boldsymbol{u}$ is the vector of displacement, $p$ is the pore-pressure, $\boldsymbol{b}$ is the body force vector, $\rho$ is the total density of the mixture, $\rho_{f}$ is the density of the fluid, $n$ is the porosity, $\bar{k}=k /\left(\rho_{f} g\right), k$ is the permeability coefficient generally used in soil mechanics, $g$ is the gravity acceleration, $K_{f}$ is the bulk modulus of the pore fluid. $\sigma^{\prime}$ is the vector form of the effective stress defined as $\sigma^{\prime}=\boldsymbol{\sigma}-\boldsymbol{m} p$ where $\sigma$ is the 
total stress, $\nabla$ is the gradient operator, $S$ is the strain operator and the vector $\boldsymbol{m}$ in the 2D case take the forms $\boldsymbol{m}^{T}=\left[\begin{array}{lll}1 & 1 & 0\end{array}\right]$. The equations have to be supplemented by a constitutive law for the soil skeleton linking $\boldsymbol{\sigma}^{\prime}$ and the strain $\boldsymbol{\varepsilon}=\boldsymbol{S u}$. For a rateindependent material, it can be expressed as $d \boldsymbol{\sigma}^{\prime}=\boldsymbol{D}_{T} d \boldsymbol{\varepsilon}$.

We now approximate the governing equations in time using the generalized Newmark scheme[8], i.e. applications of GN22 to the displacement and GN11 to the pore-pressure. Thus, the total momentum balance equation is written at $t=t_{n+1}$ as

$$
\mathbf{S}^{T}\left[\boldsymbol{\sigma}_{n+1}^{\prime}-\mathbf{m}\left(p_{n+1}^{p}+\dot{p}_{n+1} \theta \Delta t\right)\right]+\rho \mathbf{b}_{n+1}-\rho \ddot{\mathbf{u}}_{n+1}=0 .
$$

where $p_{n+1}^{p}=p_{n}+(1-\theta) \Delta t \dot{p}_{n}$. The staggered algorithm (or operator-splitting method) can be performed using an intermediate acceleration $\ddot{\boldsymbol{u}}_{n+1}^{*}$. Thus, equation (3) is approximated with the predicted value $\boldsymbol{S}^{T} \boldsymbol{\sigma}_{n+1}^{\prime p}$ and can be split into two parts as

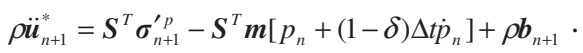

and

$$
\rho \ddot{\boldsymbol{u}}_{n+1}=\rho \ddot{\boldsymbol{u}}_{n+1}^{*}-\boldsymbol{S}^{T} \boldsymbol{m}\left[(\delta-\theta) \Delta t \dot{p}_{n}+\dot{p}_{n+1} \theta \Delta t\right] .
$$

Similarly, the conservation equation of pore-fluid flow is written at $t=t_{n+1}$ as

$$
\boldsymbol{m}^{T} \boldsymbol{S}\left(\dot{\boldsymbol{u}}_{n+1}^{p}+\ddot{\boldsymbol{u}}_{n+1} \gamma \Delta t\right)-\nabla^{T} \bar{k} \nabla\left(p_{n+1}^{p}+\dot{p}_{n+1} \theta \Delta t\right)+\frac{n}{K_{f}} \dot{p}_{n+1}+\nabla^{T} \bar{k} \rho_{f} \boldsymbol{b}_{n+1}=0
$$

Substituting equation (5) into equation (6) leads to

$$
\begin{array}{r}
\boldsymbol{m}^{T} \boldsymbol{S}\left\{\dot{\boldsymbol{u}}_{n+1}^{p}+\ddot{\boldsymbol{u}}_{n+1}^{*} \gamma \Delta t-\frac{1}{\rho} \boldsymbol{S}^{T} \boldsymbol{m}\left[(\delta-\theta) \Delta t \dot{p}_{n}+\dot{p}_{n+1} \theta \Delta t\right] \gamma \Delta t\right\} \\
-\nabla^{T} \bar{k} \nabla\left(p_{n+1}^{p}+\dot{p}_{n+1} \theta \Delta t\right)+\frac{n}{K_{f}} \dot{p}_{n+1}+\nabla^{T} \bar{k} \rho_{f} \boldsymbol{b}_{n+1}=0 .
\end{array}
$$

Performing the spatial discretisation and applying the standard Galerkin procedure to equations (4), (7) and (3) result to the following algebraic equations (for a linear elastic problem)

$$
\begin{gathered}
\boldsymbol{M} \ddot{\overline{\boldsymbol{u}}}_{n+1}^{*}=\left(\boldsymbol{f}_{s}^{*}\right)_{n+1}-\boldsymbol{K} \overline{\boldsymbol{u}}_{n+1}^{p}+\boldsymbol{Q}\left[\overline{\boldsymbol{p}}_{n}+(1-\delta) \Delta \dot{\overline{\bar{p}}}_{n}\right] . \\
\left(\mathbf{C}+\mathbf{H} \theta \Delta t+\mathbf{H}^{*} \theta \gamma \Delta t^{2}\right) \dot{\overline{\mathbf{p}}}_{n+1}=\left(\mathbf{f}_{p}\right)_{n+1}-\mathbf{H}^{*}(\delta-\theta) \gamma \Delta t^{2} \dot{\overline{\mathbf{p}}}_{n}-\mathbf{Q}^{T}\left(\dot{\overline{\mathbf{u}}}_{n+1}^{p}+\ddot{\mathbf{u}}_{n+1}^{*} \gamma \Delta t\right)-\mathbf{H} \overline{\mathbf{p}}_{n+1}^{p} . \\
\left(\boldsymbol{M}+\boldsymbol{K} \beta \Delta t^{2}\right) \ddot{\overline{\boldsymbol{u}}}_{n+1}=\left(\boldsymbol{f}_{s}\right)_{n+1}-\boldsymbol{K} \overline{\boldsymbol{u}}_{n+1}^{p}+\boldsymbol{Q}\left(\overline{\boldsymbol{p}}_{n+1}^{p}+\theta \Delta \dot{\overline{\boldsymbol{p}}}_{n+1}\right) .
\end{gathered}
$$

where

$$
\boldsymbol{H}^{*}=\int_{\Omega}\left(\nabla \boldsymbol{N}_{p}\right)^{T} \frac{1}{\rho}\left(\nabla \boldsymbol{N}_{p}\right) d \Omega
$$


As already explained in Ref. [6], the optimal elemental values of the splitting factor $\delta$ can be determined by $\delta=\delta_{0}\left(\frac{\Delta t_{\text {crit }}}{\Delta t}\right)^{2}\left(0<\delta \leq 1.0,1 / 2 \leq \delta_{0} \leq 1.0\right)$, where $\Delta t_{\text {crit }}$ is the critical time step length for the direct explicit-implicit algorithm ${ }^{[6]}$.

The so-called direct $\alpha$-method is referred to as direct modification resulting in steady state approximation which avoids mixed form restriction. Considering the presence of the pore pressure variable in first order, the divergence of the time derivative of the momentum equation should be added to the conservation equation of pore-fluid flow. The momentum balance equation (1) and its discrete form will remain unchanged. However, the conservation equation of pore-fluid flow (2) is now modified by the addition of a proportion $\alpha$ of the divergence of the time derivative of the momentum balance equation. We write thus

$$
\mathbf{m}^{T} \mathbf{S} \dot{\mathbf{u}}-\nabla^{T} \bar{k} \nabla p+\frac{n}{K_{f}} \dot{p}+\nabla^{T} \bar{k} \rho_{f} \mathbf{b}+\alpha \nabla^{T} \frac{d}{d t}\left[\mathbf{S}^{T}\left(\boldsymbol{\sigma}^{\prime}-\mathbf{m} p\right)+\rho \mathbf{b}-\rho \ddot{\mathbf{u}}\right]=0 .
$$

Applying equation (2) into equation (11), and neglecting the time derivative of the body force and the second-order time derivatives of pressure, we obtain

$$
\mathbf{m}^{T} \mathbf{S} \dot{\mathbf{u}}-\nabla^{T} \bar{k} \nabla p+\frac{n}{K_{f}} \dot{p}+\nabla^{T} \bar{k} \rho_{f} \mathbf{b}+\alpha \nabla^{T}\left(\mathbf{S}^{T} \dot{\boldsymbol{\sigma}}^{\prime}\right)-\alpha \nabla^{T} \nabla \dot{p}=0
$$

After the spatial Galerkin discretization, the total momentum balance equation and equations (12) become (for linear elasticity)

$$
\begin{gathered}
M \ddot{\bar{u}}+K \bar{u}-Q \bar{p}=\boldsymbol{f}_{s} . \\
\left(C+\tilde{\boldsymbol{H}}^{*}\right) \dot{\overline{\boldsymbol{p}}}+\boldsymbol{H} \overline{\boldsymbol{p}}+\boldsymbol{Q}^{T} \dot{\bar{u}}-\boldsymbol{L} \dot{\bar{u}}=\tilde{\boldsymbol{f}}_{p} .
\end{gathered}
$$

where

$$
\tilde{\boldsymbol{H}}^{*}=\int_{\Omega}\left(\nabla \boldsymbol{N}_{p}\right)^{T} \alpha\left(\nabla \boldsymbol{N}_{p}\right) d \Omega \quad \boldsymbol{L}=\int_{\Omega}\left(\nabla \boldsymbol{N}_{p}\right)^{T} \alpha \boldsymbol{S}^{T} \boldsymbol{D} \boldsymbol{B} d \Omega
$$

Again as already demonstrated in Ref. [6], the best solutions can be obtained with the selection of $\alpha$ value from $\alpha=\alpha_{0} \Delta t_{\text {crit }}^{2} / \rho\left(1 / 4 \leq \alpha_{0} \leq 1 / 2\right)$.

\section{Numerical Examples}

Several examples were given in Ref. [6] concerning with the solution of transient problems as well as of the final steady states obtained by the staggered implicitimplicit algorithm and direct $\alpha$-method. Those were mainly used for modeling elastic porous media on uniform and non-uniform meshes subjected to a surface step loading. The space does not permit here the full description of the performance of the proposed stabilization method in all numerical examples. Due to the significance of the success of the proposed procedures for the cases with cyclic loading, two numerical examples are presented here for a soil column and a soil foundation under a surface cyclic loading. 


\subsection{Example 1: An Elastic Soil Column Subjected to a Surface Cyclic Loading}

First, a simple example is presented to demonstrate the performance of the proposed stabilization techniques described in Section 2. This is a soil column subjected to a surface cyclic loading given by $q=1.0+0.5 \sin (2.0 t) \mathrm{kPa}$. Boundary conditions are sketched in Fig. 1. The material has been assumed isotropically elastic, with the following properties: $E=2.0 \times 10^{7} \mathrm{~N} / \mathrm{m}^{2}, \quad v=0.2, \rho_{s}=2.0 \times 10^{3} \mathrm{~kg} / \mathrm{m}^{3}, \rho_{f}=1.0 \times 10^{3} \mathrm{~kg} / \mathrm{m}^{3}$, $K_{f}=\infty, n=0.3$. Three different permeabilities of $k=0.0, k=1.0 \times 10^{-6} \mathrm{~m} / \mathrm{s}$ and $k=1.0 \times 10^{-4} \mathrm{~m} / \mathrm{s}$ are considered. For each permeability, five analyses are carried out by using various algorithms as the following: 1 - direct implicit algorithm, 10 4/4 node elements; 2 - direct implicit algorithm, 10 8/4 noded elements; 3 - staggered implicitimplicit algorithm, 10 4/4 nodded elements; 4 - direct $\alpha$-method, 10 4/4 noded elements; 5 - direct implicit algorithm, 100 8/4 noded elements. Elements with nonuniform interpolation, i.e. biquadratic for $\mathrm{u}$ and bilinear for $\mathrm{p}$ (8/4 noded elements) are considered to be acceptable. The computed results obtained using such elements can be used as the basis of comparison.

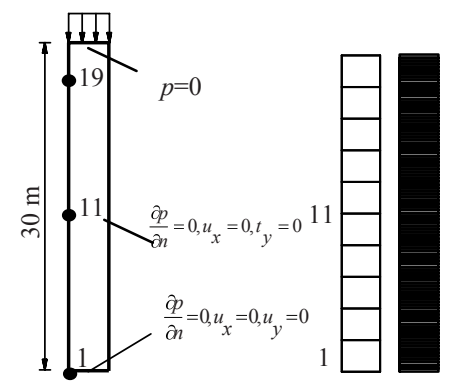

Fig. 1. A soil column subjected to a surface cyclic loading

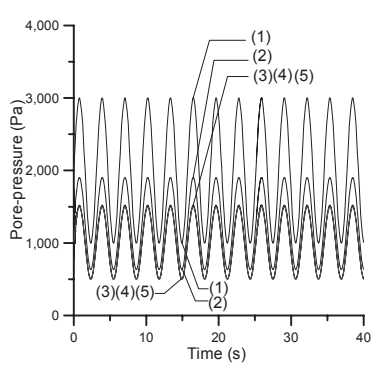

(a)

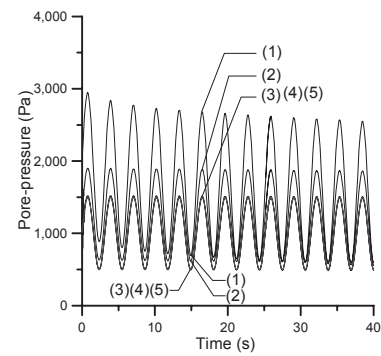

(b)

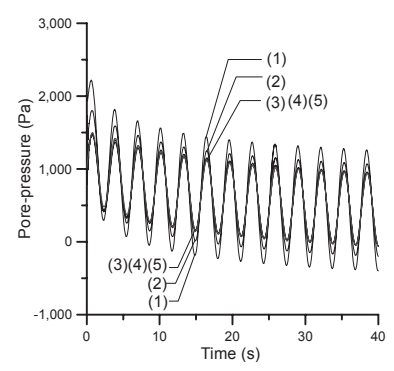

(c)

Fig. 2. Solutions for example 1 using various algorithms (Node 19): (a) $k=0.0$; (b) $k=1.0 \times 10^{-6} \mathrm{~m} / \mathrm{s}$; (c) $k=1.0 \times 10^{-4} \mathrm{~m} / \mathrm{s}$ 
It is observed from Fig. 2(a), (b) and (c) that the solutions obtained by using direct implicit algorithm with equal interpolation (i.e. 4/4 noded element) overestimate the initial pore-pressure significantly, especially for the cases of low permeability. The results can be improved when the proposed direct $\alpha$-method is introduced. As far as the results of pore-pressure are concerned, the proposed procedure performs even better than mixed elements with non-uniform interpolation (i.e. 8/4 noded element) for a mesh composed of 10 elements. In fact, the computational results of porepressure obtained by using the stabilization $\alpha$-method and the staggered implicitimplicit algorithm agree well with those from 100 8/4 noded elements.

\subsection{Example 2: An Elastic Soil Foundation Subjected to a Surface Cyclic Loading}

Next, to further demonstrate the performance of the proposed stabilization methods, numerical investigations of a real two-dimensional example with uniform and nonuniform meshes are carried out. This is an elastic foundation again with a surface cyclic loading same as that in example 1 . The boundary conditions are sketched in the Fig. 3(a). The material properties are identical to those in example 1.

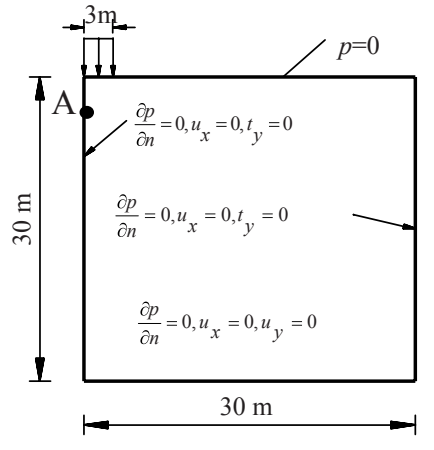

(a)

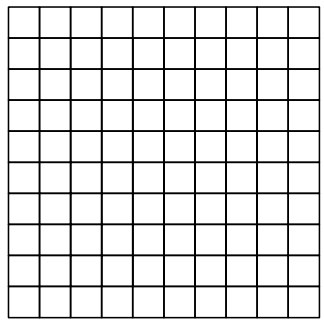

(b)

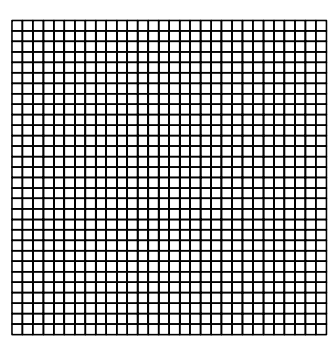

(c)

Fig. 3. A soil foundation subjected to a surface cyclic loading: (a) geometry and boundary conditions; (b) uniform mesh - 100 elements; (c) uniform mesh - 900 elements

\subsubsection{Uniform Meshes (Fig. 3b, 3c)}

Two uniform meshes used in the analyses consist of 100 or 900 quadrilateral elements as depicted in Fig. 3(b) and Fig. 3(c). Again, three different permeabilities are studied with comparisons of five different algorithms similar to those already mentioned in example 1, except 100 elements used for the algorithm Nos. 1, 2, 3, 4 and 900 elements used for the algorithm No. 5. The numerical results obtained by using various algorithms are shown in Figs. 4(a), (b) and (c). As expected, similar conclusions can be made with the excellent performance of the proposed stabilization method. 


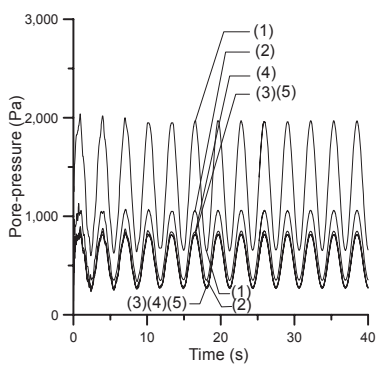

(a)

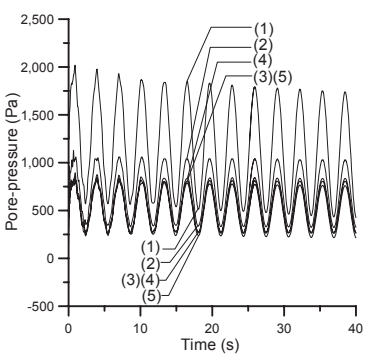

(b)

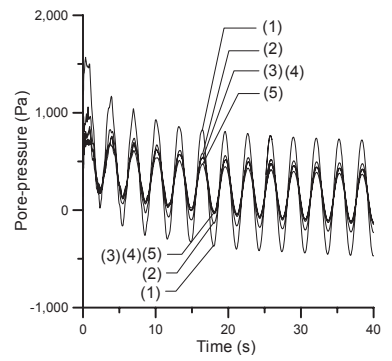

(c)

Fig. 4. Solutions for example 2 using various algorithms with uniform meshes (Node A): (a) $k=0.0 ;$ (b) $k=1.0 \times 10^{-6} \mathrm{~m} / \mathrm{s}$; (c) $k=1.0 \times 10^{-4} \mathrm{~m} / \mathrm{s}$

\subsubsection{Non-uniform Mesh (Fig. 5)}

As already demonstrated in Ref. [6], the staggered implicit-implicit algorithm with a global splitting factor $\delta$ gives us a significant oscillation for non-uniform mesh since only global time step can be used for transient problems. The computed results of pore-pressure can be improved when local splitting factors $\delta$ are introduced. The nonuniform mesh used for the analysis is shown in Fig. 5 and it consists of only $4 / 4$

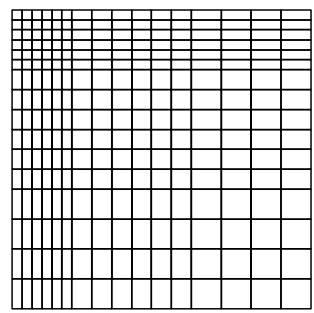

Fig. 5. A non-uniform mesh used for example 2

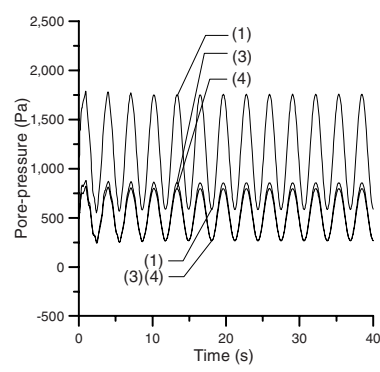

(a)

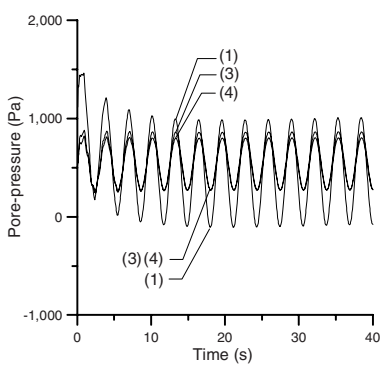

(b)

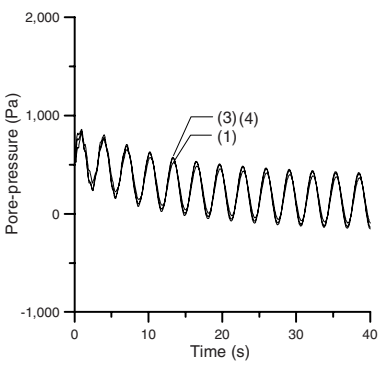

(c)

Fig. 6. Solutions for example 2 using various algorithms with non-uniform mesh (Node A): (a) $k=0.0$; (b) $k=1.0 \times 10^{-6} \mathrm{~m} / \mathrm{s}$; (c) $k=1.0 \times 10^{-4} \mathrm{~m} / \mathrm{s}$ 
noded elements. Fig. 6(a), 6(b) and 6(c) present the comparisons of the computed pore-pressure obtained by using three different algorithms ( 1 - direct implicit algorithm; 3 - staggered implicit-implicit algorithm with local $\delta ; 4$ - direct $\alpha$ method). Again, the results of pore-pressure can be improved when the proposed stabilization method is introduced, especially for the cases with low permeabilities.

\section{Conclusions}

In this paper, two classes of the stabilization methods proposed previously by the authors for saturated soil dynamics in the limit of nearly incompressible pore-fluid and small permeability has been briefly reviewed. Attention is then paid to the performance of the proposed procedures in the problems subjected to cyclic loading, and two numerical examples have been presented. The numerical results have shown a good performance of the proposed procedures. It must be pointed out here that the extension of the stabilized procedures to the elasto-plastic problems is of significantly importance for practical purpose. Further research in the elasto-plastic analysis with a more complicated constitutive model based on the concept of bounding-surface plasticity is currently underway. It must be pointed out that the success in elastoplastic problems is due to not only the introduction of the proposed stabilization method but also the use of adaptive remeshing or other suitable techniques such as the assumed strain method, etc. The incompressible-undrained issue can be solved using the proposed stabilization method with the elements of equal linear interpolation. However, such elements still behave poorly for the bending dominated problems, which are important in the calculations involving strain localization. It must be emphasized that the difficulties remaining for the elasto-plastic problems should not underestimate the importance in the determination of stabilization parameters. Optimal elemental parameters can provide the best solutions for the pore-pressure, which are obviously essential to the success not only in elastic problems but also for elasto-plastic cases.

Acknowledgments. The author wishes to thank the financial support provided by the National Natural Science Foundation of China under research grant No. 10272083.

\section{References}

1. Zienkiewicz OC, Chang CT, Bettess P. Drained, undrained, consolidating and dynamic behaviour assumptions in soils; Limits of validity. Geotechnique, 1980, 30:385-395.

2. Zienkiewicz OC, Shiomi T. Dynamic behaviour of saturated porous media: the generalised Biot formulation and Its numerical solution. Int J Numer Analyt Meth Geomech, 1984, 8:7196.

3. Zienkiewicz OC, Chan AHC, Pastor M, Paul DK, Shiomi T. Static and dynamic behaviour of soils: a rational approach to quantitative solutions, Part I: Fully saturated problems. Proc $R$ Soc London, 1990, A429:285-309.

4. Zienkiewicz OC, Huang M, Wu J, Wu S. A new algorithm for coupled soil-pore fluid problem. Shock and Vibration, 1993, 1:3-14. 
5. Huang M, Wu S, Zienkiewicz OC. Incompressible or nearly incompressible soil dynamic behaviour - a new staggered algorithm to circumvent restrictions of mixed formulation. Soil Dynamics and Earthquake Engineering, 2001, 21:169-179.

6. Huang M, Yue ZQ, Tham LG, Zienkiewicz OC. On the stable finite element procedures for dynamic problems of saturated porous media. Int J Numer Meth Engng, 2004, 61:14211450.

7. Mira P, Pastor M, Li T, Liu X. A new stabilized enhanced strain element with equal order of interpolation for soil consolidation problems. Comput Methods Appl Mech Engrg, 2003, 192:4257-4277.

8. Zienkiewicz O C, Taylor R L. The Finite Element Method, 5th Edition. Oxford: Butterworth Heinemann, 2000. 